\title{
Eficácia da toxina botulínica tipo-A associada a fisioterapia em uma criança hemiplégica espástica
}

\section{Effectiveness of BTA and physiotherapy in treating a children with hemeplegic-spastic cerebral palsy}

Resende, C. M. G. ${ }^{1}$; Nascimento, V. F. do'; Leite, J. M. R. S. ${ }^{2}$

\section{RESUMO}

Introdução: Paralisia cerebral (PC) caracteriza um grupo de distúrbios cerebrais de caráter estacionário, que podem se manifestar clinicamente como hemiparesia e espasticidade. A toxina botulínica tipo A (TBA) atua na junção neuromuscular, bloqueando a liberação da acetilcolina na placa motora e assim, proporciona um relaxamento maior da musculatura, sendo eficaz no tratamento da paralisia cerebral, principalmente se associada a um tratamento fisioterápico através da cinesioterapia, hidroterapia e o uso de órteses. Objetivo: Este estudo visa analisar a eficácia da TBA e fisioterapia em uma criança com paralisia cerebral padrão hemiparético-espástico. Material e método: Participou deste estudo uma criança hemiplégica espástica, submetida ao tratamento fisioterápico. A primeira avaliação, antes da aplicação da TBA, a segunda, após 15 dias da aplicação, em que foi realizado o tratamento fisioterápico associado ao uso de órtese. Em cada avaliação foi verificada a amplitude de movimento, a marcha e a espasticidade. Resultados: Na avaliação da goniometria, verificou-se uma melhora da mobilidade do membro inferior direito. A marcha também melhorou significantemente e a espasticidade diminuiu. Conclusão: A TBA foi eficaz e segura em nosso paciente e em associação com o tratamento fisioterápico, proporcionou maior independência, melhora da marcha e da qualidade de vida de nosso paciente com PC hemiplégica.

Unitermos: Paralisia cerebral, Hemiplegia, Espasticidade, Toxina botulínica tipo A.

Citação: Resende CMG, Nascimento VF, Leite JMRS. Eficácia da toxina botulínica tipo-A associada a fisioterapia em uma criança hemiplégica espástica. Rev Neurociencias 2005; 13(1):017-020.

\section{SUMMARY}

Context: Cerebral Palsy characterizes a group of cerebral disturbances of stationary character. The botulinum toxin A (BTA) acts in the neuromuscular junction, blocking acetylcholine liberation providing a larger relaxation of the musculature. It is important to accomplish a physiotherapeutic treatment through kinetics, hydrotherapy and orthesis to reach a better improvement to the patient. Objective: This study analyzes the effectiveness of BTA in a child with cerebral palsy submitted to the physiotherapeutic treatment. Material and method: a child with cerebral palsy was clinically evaluated and treated with BTA andphysiotherapy. The measures were done pre treatment and after 15 days post BTA.. In each evaluation it was verified the movement width, the march and the spasticity. Results: The child presented movement

Trabalho realizado: Centro Universitário de Lavras - UNILAVRAS

1 - Acadêmicas do curso de Fisioterapia do Centro Universitário de Lavras - UNILAVRAS;

2 - Mestre e doutorando da UNIFESP/EPM, professora do Centro Universitário de Lavras - UNILAVRAS. 
amplitude and clinical improvement compared to pre treatment evaluation. The spasticity also improved. Conclusion: Botulinum toxin $\mathrm{A}$ is an effective and safe method, that in association with the physiotherapy were associated to a larger independence of child with cerebral palsy.

\section{Keywords: Cerebral palsy, Hemiplegia, Spasticity, Botulinum toxin A.}

Citation: Resende CMG, Nascimento VF, Leite JMRS. Effectiveness of BTA and physiotherapy in treating a children with hemeplegic - spastic cerebral palsy. Rev Neurociencias 2005; 13(1):017-020.

\section{INTRODUÇÃO}

Paralisia cerebral (PC) constitui um grupo de distúrbios cerebrais de caráter estacionário, que são devidos a uma lesão ou anomalias do desenvolvimento ocorridas durante a vida fetal ou durante os primeiros meses de vida.

A PC pode ser classificada, de acordo com os sinais clínicos em cinco grupos: atáxica, hipotônica, atetósica, espástica (tetraplegia, diplegia, hemiplegia) e mista. A forma espástica é a mais comum, pode ser definida como uma resistência ao estiramento passivo de um músculo ou de um grupo muscular (hipertonia), devido acometimento do neurônio motor superior Sistema Nervoso Central $(\mathrm{SNC})^{1-2}$.

A hemiplegia espástica afeta os membros de um lado do corpo, o lado comprometido é o oposto ao lado cerebral lesado. Há hipertonia em flexão do membro superior e em extensão do membro inferi$\mathrm{or}^{3}$. O pé assume posição equinovaro, o que dificulta a marcha ${ }^{4}$.

A espasticidade é um dos maiores obstáculos para a reabilitação neurológica ${ }^{5}$, o que suscitou o uso da toxina botulínica tipo A (TBA - derivada da bactéria gram negativa e anaeróbica Clostridium botulinium) ${ }^{6}$, para o tratamento desta condição clínica.

São conhecidos 8 sorotipos de toxinas, das quais 7 são neurotóxicas. A toxina botulínica tipo- $A$ é a mais potente. Trata- se de uma proteína polipeptídica composta por duas cadeias, uma leve e outra pesada, ligadas por uma ponte dissulfídica ${ }^{7}$. A TBA é uma neurotoxina que atua na junção neuromuscular, bloqueando a liberação da acetilcolina na placa motora, e proporcionando um relaxamento maior da musculatura, permite um alongamento maior dos músculos injetados e meIhora o padrão da marcha8.

As medidas fisioterápicas, por meio da cinesioterapia, através de alongamentos suaves, fortalecimento dos músculos e posicionamento adequado visam tornar os músculos menos responsivos aos estímulos nervosos alterados ${ }^{9}$.
A hidroterapia alia os efeitos benéficos da fisioterapia de solo às propriedades físicas da água, como o empuxo e a pressão hidrostática, que tornam o movimento mais fácil de ser realizado dentro da água, auxilia no fortalecimento e tem efeito relaxante adicional proporcionado pelo calor ${ }^{10-13}$.

O uso de órteses para a manutenção do posicionamento articular adequado, é de fundamental importância no tratamento da espasticidade e após aplicação da TBA,7-8. Estas, posicionam os músculos em determinado grau de alongamento evitando que os mesmos entrem em retração e podem auxiliar na função, melhorando o padrão da marcha $^{8,14}$.

A utilização da TBA neste trabalho torna-se de fundamental importância, pois, promove uma diminuição da espasticidade melhorando o padrão da marcha favorecendo, assim, a reeducação funcional e muscular do membro acometido.

\section{MATERIAL E MÉTODO}

Participou deste estudo uma criança, do sexo masculino, com idade cronológica de 5 anos, compatível com a idade motora, com diagnóstico clínico de Paralisia Cerebral e diagnóstico fisioterapêutico de hemiplegia espástica direita.

O estudo foi realizado na Clínica de Fisioterapia Risoleta Neves, que pertence ao Centro Universitário de Lavras-UNILAVRAS. Antes de iniciar o estudo, o responsável pela criança assinou um termo de consentimento livre e esclarecido .

Os materiais utilizados na piscina constituíamse basicamente de um tablado, um tapete de flutuação, um estepe, flutuadores em forma de macarrão e brinquedos em geral. No solo, foram utilizados tatame, barras paralelas, rampas, escadas e bola suíça.

Para a coleta de dados foi avaliado os graus de movimento dos membros inferiores através do goniômetro da marca $\mathrm{CARCl}^{15}$. A espasticidade foi avaliada pela escala de Ashowrth Modificada, que 
gradua a espasticidade de 0-4 graus. A marcha foi avaliada através de uma ficha específica para pacientes portadores de PC hemiplégica espástica, que contém 3 domínicos ${ }^{16}$ já descritos em outra parte: o primeiro registra pontuação para cada fase da marcha (contato inicial, oscilação, suporte), o segundo registra os pontos obtidos pelo paciente no estado funcional da marcha para cada qualificador, e o terceiro classifica a marcha clinicamente a partir da pontuação obtida anteriormente em marcha normal (0 pontos); marcha com comprometimento mínimo, (1 a 14 pontos); marcha com comprometimento significante (15 a 30 pontos) e marcha com comprometimento limitante (>30 pontos) .

A criança foi submetida a duas avaliações, a primeira antes da aplicação da TBA e a segunda após 15 dias em que foi realizado o tratamento fisioterápico associado ao uso de órtese. O tratamento fisioterápico foi realizado no solo às terças e quintas e hidroterapia às segundas, quartas e sextas, com duração da sessão de 45 minutos em todas as sessões.

A TBA foi aplicada nos músculos tibial posterior e tríceps sural. Após 48 horas da aplicação, iniciou-se o tratamento fisioterápico e a mãe da criança foi orientada a realizar exercícios domiciliares propostos pelo fisioterapeuta. Os dados foram analisados através do teste $t$ de Student.

\section{RESULTADOS}

A tabela 1 mostra a análise da goniometria do membro inferior direito realizada antes e após a aplicação da TBA. Os valores goniométricos relativos ao membro inferior direito apresentaram melhora significante após o tratamento $(p<$ $0,006)$. Os indicadores clínico-funcionais da marcha, obtidos através da Ficha de Avaliação da Marcha Hemi-plégico-espástica (FAMHE) ${ }^{16}$, também evidenciaram melhora estatisticamente significante $(p<0.04)$. A espasticidade foi avaliada pela Escala de Ashworth Modificada, a primeira avaliação resultou em grau 2, o que significa que ocorreu um aumento do tônus acentuado e, a segunda avaliação resultou em grau 1. A tabela 2 mostra a pontuaçaõ abtida através da "Ficha de Avaliação da Marcha Hemiplégica Espástica", antes e após a aplicação da TBA.

A criança incluída neste estudo, encontra-se com características comuns às citadas por Diament e $\mathrm{Cypel}^{4}$, evidenciando hipertonia em flexão no mem-

\begin{tabular}{|l|l|r|r|}
\hline \multirow{2}{*}{} & Pré TBA & Pós TBA \\
\hline \multirow{4}{*}{ Quadril } & Flexão & $90^{\circ}$ & $100^{\circ}$ \\
\cline { 2 - 4 } & Extensão & $5^{\circ}$ & $8^{\circ}$ \\
\cline { 2 - 4 } & Abdução & $40^{\circ}$ & $42^{\circ}$ \\
\cline { 2 - 4 } & Adução & $10^{\circ}$ & $12^{\circ}$ \\
\cline { 2 - 4 } & Rot. Int. & $35^{\circ}$ & $35^{\circ}$ \\
\cline { 2 - 4 } & Rot. Ext. & $41^{\circ}$ & $41^{\circ}$ \\
\hline \multirow{4}{*}{ Joelho } & Flexão & $120^{\circ}$ & $130^{\circ}$ \\
\hline \multirow{4}{*}{ Tornozelo } & Dorsiflexão & $10^{\circ}$ & $15^{\circ}$ \\
\cline { 2 - 4 } & Flexão plantar & $60^{\circ}$ & $48^{\circ}$ \\
\cline { 2 - 4 } & Inversão & $46^{\circ}$ & $40^{\circ}$ \\
\cline { 2 - 4 } & Eversão & $18^{\circ}$ & $18^{\circ}$ \\
\hline
\end{tabular}

Tabela 1. Análise da "Goniometria" do membro inferior direito comparando-se a $1^{\underline{a}}$ com a $2^{\underline{a}}$ avaliação $(p=0,0066)$.

\begin{tabular}{|l|c|c|}
\hline & Pré TBA & Pós TBA \\
\hline Pé & 6 & 4 \\
\hline Joelho & 8 & 6 \\
\hline Artelhos & 2 & 2 \\
\hline Pelve & 4 & 2 \\
\hline Tronco & 4 & 4 \\
\hline Flexão da coxa & 2 & 2 \\
\hline Extensão da coxa & 2 & 2 \\
\hline Arrastamento do membro & 1 & 0 \\
\hline TOTAL & $\mathbf{2 9}$ & $\mathbf{2 0}$ \\
\hline
\end{tabular}

Tabela 2. Pontuação da Marcha comparando-se a $1^{\underline{a}} \mathrm{com}$ a $2^{\underline{a}}$ avaliação $(p=0,0412)$.

bro superior e extensão no membro inferior, pé eqüinovaro, dificultando assim sua marcha.

A toxina pode ser considerada um método terapêutico seguro e eficaz para o tratamento da espasticidade, quando os métodos tradicionais faIham $^{5}$. O que foi verificado neste estudo, pois após 48 horas da aplicação da TBA, pode-se verificar o início do seu efeito, tendo ocorrido melhora da mobilidade articular do tornozelo em todas as amplitudes. Não foi verificado presença de efeitos colaterais nos primeiros 15 dias.

O estudo realizado avaliou o efeito da TBA em apenas 15 dias $^{9}$. O autor relata que a toxina atinge seu efeito máximo em mais ou menos 15 dias após a aplicação. Outros estudos ${ }^{6}$ relatam que a TBA atinge o seu efeito máximo em 1 a 4 semanas, o que indica que a toxina poderia ainda não ter atingido seu efeito máximo em nosso paciente quando obtivemos os dados para este estudo. 
A TBA inibe a espasticidade, facilita os movimentos, proporcionando uma melhora do padrão da marcha ${ }^{8,17}$. O que pode ser evidenciado ao comparar a primeira com a segunda avaliação, houve uma melhora da marcha, uma melhora da mobilidade articular do membro inferior direito e uma melhora da espasticidade, sendo este um resultado estatisticamente significante.

A mãe da criança teve um papel importante para a realização deste estudo, por colaborar em todo o período. Com pontualidade, presença e disponibilidade na grande maioria das sessões. A criança dificultou o procedimento do tratamento fisioterápico por ser hiperativa e super protegida pela mãe.

Após a aplicação da TBA, a conquista em termos de realização de exercícios e os resultados adquiridos neste trabalho, estimularam a criança a ter uma maior satisfação, alegria e prazer a cada sessão, diminuindo sua hiperatividade que antes da aplicação, era um fator limitante na realização do tratamento.

\section{CONCLUSÃO}

O uso da toxina botulínica tipo A veio trazer um novo rumo para a reabilitação neurológica, oferecendo ganhos antes impossíveis, tendo-se demonstrado neste estudo e em outros da literatura um método de tratamento da espasticidade eficaz e seguro, que em associação com o tratamento fisioterápico, proporciona maior independência, melhora da marcha e da qualidade de vida de crianças portadoras de paralisia cerebral hemiplégico-espástica.

\section{REFERÊNCIAS BIBLIOGRÁFICAS}

1. Chagas PSC, Mancini MC, Barbosa AP, Silva, PTG. Análise das Intervenções Utilizadas para promoção da marcha em crianças portadoras de Paralisia Cerebral - Uma Revisão Sistemática de Literatura. Rev Bras Fisioter 2004; 18(2)

2. Mancini MC, et al. Comparação do Desempenho de atividades funcionais em crianças com desenvolvimento normal e crianças com Paralisia Cerebral. Arq Neuropsiquiatr 2002; $60(2 B): 446-452$

3. Costa M. Incapacidade Motora Cerebral "Paralisia Cerebral". Disponível em: www.fisioweb.com.br/ Acessado em: 18/08/04.

4. Diament A, Cypel S. Neurologia Infantil. São Paulo: Atheneu, 1996.

5. Teive, HAG, Zonta M., Kumagai Y. Tratamento da espasticidade: uma atualização. Arq Neuropsiquiatr 1998; 56(4):852-858.

6. Siqueira B. A utilização da toxina botulínica do tipo A ( BOTOX?) no tratamento da espasticidade. Disponível em: www.fisioweb.com.br / Acessado em: 18/08/04.

7. Martins SMQ, Guidice AVD. Um novo auxílio para tratar Paralisia Cerebral. Rev Fisio \& Terapia 2001; 5(28).

8. Lima CLA, Fonseca LF. Paralisia Cerebral: Neurologia, Ortopedia, reabilitação. Rio de Janeiro: Guanabara Koogan, 2004.
9. Leite JMRS, Prado GF. Paralisia Cerebral, aspectos fisioterapêuticos e clínicos. Rev Neuroci 2004; 12(1): 41-45.

10. Campion MR. Hidroterapia e Princípios e Práticas. São Paulo: Ed. Manole, 2000.

11. Caromano FA, et al. Efeitos fisiológicos da imersão e do exercício na água. Rev Fisioter Bras 2003; 4(1)

12. Caromano FA, Ide MR. Movimento na Água. Rev Fisioter Bras 2003; 4(2).

13. Caromano FA, Nowotny JP. Princípios Físicos que Fundamentam a Hidroterapia. Rev Fisioter Bras 2002; 3(6): 394-402.

14. Gialiam HK, et al. Recomendações para o uso da toxina botulínica tipo A no tratamento da paralisia cerebral. Revista BOTOX, 1999

15. Marques AP. Manual de Goniometria. São Paulo: Manole, 1997.

16. Leite JMRS. Fisioterapia e Toxina Botulínica Tipo A no Tratamento de Seqüelas Motoras em Pacientes com Paralisia Cerebral Hemiplégico - Espástico: Ensaio Clínico Randomizado. Tese de Mestrado Profissionalizante, Universidade Federal de São Paulo (UNIFESP), Programa de Pós-Graduação em Medicina Interna e Terapêutica, 2002, São Paulo.

17. Gianni MAC. Tratamento da espasticidade. Rev Reabil 200; 7:33-39. 